\title{
Procrastinación académica en el alumnado universitario no tradicional
}

\section{Angélica Garzón-Umerenkova ${ }^{1}$, Javier Gil-Flores ${ }^{2}$}

\author{
${ }^{1}$ Facultad de Psicología. Universidad El Bosque. Bogotá, Colombia \\ ${ }^{2}$ Departamento MIDE. Universidad de Sevilla
}

\section{Colombia / España}

Correspondencia: Javier Gil Flores. Facultad de Ciencias de la Educación. C/Pirotecnia, s/n. 41003-Sevilla. España. E-mail: jflores@us.es 


\section{Resumen}

Introducción. La procrastinación es la acción de retrasar la realización de tareas necesarias, hasta el extremo de generar malestar y perjuicios a los individuos que la protagonizan. La presencia de alumnado no tradicional en las universidades se ha incrementado notablemente en las últimas décadas. Este tipo de estudiantes encuentra dificultades en sus estudios, las cuales podrían propiciar una mayor incidencia de las conductas procrastinadoras. Para comprobarlo, comparamos los niveles de procrastinación y motivos para procrastinar entre estudiantes tradicionales y no tradicionales.

Método. Utilizamos una muestra de 479 estudiantes colombianos. Los niveles de procrastinación y motivos para procrastinar fueron medidos a partir de la Procrastination Assessment Scale - Students (PASS). En el análisis de datos se utilizaron estadísticos descriptivos, análisis factorial exploratorio, prueba t para muestras independientes y d de Cohen.

Resultados. Los estudiantes no tradicionales han presentado niveles de procrastinación superiores $(t=4,412 ; p<0,001 ; d=0,459)$, aunque los motivos para procrastinar no difieren de los aportados por estudiantes tradicionales. El carácter de estudiante no tradicional ha resultado ser una variable relevante en la explicación de la procrastinación académica.

Discusión y conclusiones. A partir de estos resultados, se deriva la conveniencia de ofrecer una orientación académica al alumnado no tradicional, con el fin de tratar la procrastinación y reducir así posibles riesgos de bajo rendimiento o abandono en este colectivo estudiantil.

Palabras Clave: procrastinación, estudiantes no tradicionales, educación superior, orientación académica. 


\begin{abstract}
Introduction. Procrastination is the act of delaying necessary tasks until the extreme of experiencing discomfort and negative consequences to the individuals. The presence of nontraditional students at universities is a phenomenon which has increased remarkably over the last decades. This type of university students finds some difficulties during their grade. This could result in a higher prevalence of procrastination behaviors among nontraditional students at university. To test this, our work compares procrastination levels and reasons to procrastinate among traditional and nontraditional students
\end{abstract}

Method. A sample of 479 Colombian college students was used. Procrastination and the reasons to procrastinate are measured by the Procrastination Assessment Scale - Students $(P A S S)$. The data analysis is based on descriptive statistics, exploratory factorial analysis, $\mathrm{t}$ test for independent samples and Coohen's d.

Results. Nontraditional students show higher procrastination levels $(t=4,412 ; p<0,001$; $d=0,459$ ), although their reasons to procrastinate are similar to those of traditional students. The nontraditional student condition is a demographic variable relevant for explaining academic procrastination.

Discussion and Conclusion. These results suggest the convenience of offering academic counselling and guidance to nontraditional students, in order to attend procrastination and reducing that way low achievement or dropout risks.

Keywords: procrastination, nontraditional students, demographics, Higher Education, academic counselling. 


\section{Introducción}

\section{Procrastinación}

Dejar para más tarde o para el último momento la toma de una decisión, la realización de una actividad o el cumplimiento de una obligación es algo que, en mayor o menor medida, muchas personas han practicado alguna vez en las más diversas situaciones. A ello hace referencia el término procrastinación. Etimológicamente, esta palabra de origen latino está formada por el prefijo pro, que denota acción o movimiento hacia adelante, y crastinus, con el sentido de aquello que corresponde al día de mañana. Procrastinar significa literalmente "mover a mañana", es decir aplazar o diferir algo.

Retrasar una tarea puede ser a veces un modo de conducta adaptativa, que las personas ponen en práctica porque priorizan otras más importantes, consideran necesario disponer de más información o más recursos antes de abordarla (Ferrari, 2010). Algunos individuos la utilizan como estrategia para trabajar bajo presión y maximizar así la motivación, o bien lograr el máximo rendimiento con la menor inversión de tiempo (Schraw, Wadkins y Olafson, 2007). Tales formas de actuar responden a lo que podría denominarse una demora funcional o estratégica (Klingsieck, 2013).

Este tipo de retraso activo al abordar una tarea, cuyas consecuencias pueden ser positivas, se distancia de las conductas persistentes de aplazamiento que, aunque realizadas de manera voluntaria, resultan innecesarias e ilógicas, y llegan a interferir en la vida cotidiana representando un problema para quienes las protagonizan. Esta es la acepción habitual en el ámbito psicológico, donde el concepto de procrastinación se entiende como un fallo de autorregulación en el individuo (Pychyl y Flett, 2012; Rozental y Carlbring, 2014; Steel, 2007). La procrastinación implica aplazar el comienzo o la finalización de una tarea importante para el individuo hasta casi agotar o sobrepasar el tiempo límite fijado, e incluso de manera indefinida, aun sabiendo que lo ideal habría sido realizarla en el momento presente (Gupta, Hershey y Gaur, 2012; Rozental y Carlbring, 2013). Los procrastinadores recurrentes no solo ponen en riesgo la culminación y el éxito en las tareas afectadas, sino que pueden experimentar problemas en sus relaciones personales, estrés, ansiedad o depresión (Rozental y Carlbring, 2014). A diferencia de lo que ocurre cuando se pospone deliberadamente la ejecución de una actividad, la procrastinación va más allá del retraso en la realización de tareas, generando malestar 
en los individuos. Implica frustración, descontento del sujeto con este retraso o sentimiento de culpa (Corking, Yu y Lindt, 2011; Krause y Freund, 2014).

Numerosos estudios han analizado el porcentaje de personas implicadas en conductas procrastinadoras hasta el punto de generarles angustia y dificultades como las que acabamos de mencionar. En el meta-análisis realizado por Steel (2007) sobre este tipo de trabajos, se constataba con el paso de los años un aumento en la prevalencia de la procrastinación en la sociedad en general. Desde cifras del 4\%-5\% de adultos procrastinadores en los años setenta, se ha pasado en trabajos más recientes a porcentajes del 15\%-20\% de adultos afectados de manera crónica (Steel, 2012). En consecuencia, la procrastinación constituye un comportamiento bastante extendido en la actualidad.

En la literatura científica se han identificado una serie de factores asociados a las conductas de procrastinación. En lo que respecta a las características demográficas de los sujetos, la investigación se ha centrado en la incidencia del género y la edad (véase el meta-análisis de Steel, 2007). Los resultados muestran que los hombres procrastinan ligeramente más que las mujeres. Una posible explicación se encuentra en que los hombres tienden a presentar mayores niveles de impulsividad (Strüber, Lück y Roth, 2008) y menores niveles de autocontrol (Higgins y Tewksbury, 2006). En cuanto a la edad, el desarrollo de funciones cognitivas superiores esenciales para la autorregulación no se da hasta la adolescencia, explicando una mayor procrastinación en los sujetos más jóvenes (Rozental y Carlbring, 2014). Además, una mayor edad implica mayor experiencia y la posibilidad de que sujetos procrastinadores hayan conseguido desarrollar estrategias para evitar las demoras injustificadas. A pesar de todo, en la literatura previa, las correlaciones de género y edad con la procrastinación han venido arrojando valores pequeños, aunque significativos estadísticamente al ser calculados en muestras grandes; ello confiere un valor sustantivo limitado a tales relaciones (Ferrari, 2010).

La macroencuesta sobre población adulta de ocho países de habla inglesa repartidos por cuatro continentes, realizada por Steel y Ferrari (2013), incluyó variables demográficas que en estudios anteriores habían resultado estar asociadas a las tendencias procrastinadoras. Además de la vinculación con el género y la edad, los hallazgos de este estudio muestran también una relación con las variables estado civil y nivel de educación. Así, el perfil del procrastinador sería el de un hombre joven, soltero y con bajo nivel de formación. El tamaño 
familiar y la ubicación geográfica de su residencia no resultaron variables relevantes en la explicación de las conductas procrastinadoras.

Han sido numerosas las investigaciones sobre la relación entre procrastinación y diferentes rasgos de personalidad (Steel, 2007). Elevados niveles de neuroticismo, impulsividad, y bajos niveles de concentración, organización o autocontrol se han identificado como buenos predictores. En cambio, se ha descartado su relación con extraversión, inteligencia y aptitudes. En el ámbito de las concepciones disfuncionales de los individuos, se asocia a la procrastinación el exceso de perfeccionismo, la baja autoestima, la baja autoeficacia percibida y niveles bajos de tolerancia a la frustración (Pychyl y Flett, 2012). Otra variable estudiada ha sido la motivación del sujeto. De acuerdo al modelo de dos factores de Strunk et al. (2013), el continuo motivacional iría desde la motivación al logro a la motivación por evitación. Ambas fuentes motivacionales podrían dar lugar a que las personas procrastinen o no, pero por razones muy distintas: por logro, en la búsqueda de un mejor rendimiento o mejora de las habilidades propias, o por evitación de consecuencias indeseables. También las características de la propia tarea llevarían a procrastinar en mayor o menor medida, dependiendo del atractivo de la misma y del interés que despierta (Ferrari y Scher, 2000).

Teniendo en cuenta los hallazgos de la investigación que se habían venido desarrollando durante las últimas décadas, Balkis y Duru (2007) sitúan entre las causas más comunes de la procrastinación una pobre gestión del tiempo, sentimientos de estar abrumado, falta de motivación, falta de habilidades para la organización del trabajo, dificultades de concentración, miedo y ansiedad ante el fracaso, baja autoconfianza, problemas personales de diversa naturaleza, expectativas irreales o excesivo perfeccionismo.

\section{Procrastinación académica}

La prevalencia de las conductas procrastinadoras se dispara cuando situamos el foco en la población estudiantil. Para Rozental y Carlbring (2014), si bien la procrastinación afecta a un quinto de la población adulta, podría estar presente en la mitad de los estudiantes universitarios. De acuerdo con Day, Mensink y O’Sullivan (2000), al menos el 50\% de este alumnado procrastina de manera reiterada y problemática. Aún más, Steel y Ferrari (2013) cifraban el porcentaje de alumnado universitario que difiere tareas entre el $80 \%$ y el $95 \%$, la mayor parte de los cuales reconocen ser procratinadores. 
Los valores comentados llevan a considerar la procrastinación como un fenómeno con importante presencia en la universidad. Esperar al último día para la entrega de trabajos, diferir el estudio hasta períodos de tiempo próximos a la celebración de exámenes, o dejar la matriculación en determinadas materias para cursos académicos posteriores son prácticas ampliamente extendidas entre el alumnado universitario. En estos términos se concreta la procrastinación académica, definida como un comportamiento disfuncional que supone evitar, posponer o justificar retrasos en una tarea académica (Onwuegbuzie, 2004).

Especialmente en el ámbito educativo, la procrastinación constituye un importante problema, tanto por su alta prevalencia como por las consecuencias derivadas. Como en el resto de la población, los estudiantes procrastinadores sufren los problemas de estrés, ansiedad o sentimientos negativos a los que ya hemos hecho referencia. Además, el estudio de Sirois (2004) reveló un aumento de la intensidad de enfermedades sufridas por los estudiantes universitarios que procrastinan. Este incremento de la morbilidad se registra especialmente al aproximarse la finalización del período lectivo, momento en el que se concentran tareas anteriormente pospuestas. Han de tenerse también en cuenta las consecuencias observadas sobre el aprendizaje. Entre los estudiantes, procrastinar representa el resultado de una ineficaz gestión del tiempo, que retrasa el logro de las metas académicas y reduce el rendimiento (Ferrari y Díaz-Morales, 2014; Kim y Seo, 2015). Entre otras razones, este efecto negativo sobre el aprendizaje es debido a que los procrastinadores cometen más errores, trabajan más despacio y desatienden más las directrices de trabajo recibidas (Abbasi y Alghamdi, 2015).

\section{Alumnado universitario no tradicional}

El tipo de alumnado que cursa estudios universitarios se ha ido diversificando considerablemente respecto a épocas pasadas, a medida que se ha democratizado el acceso a la educación superior. Queda ya lejano el perfil tradicional de estudiante de nuevo ingreso, personalizado en jóvenes que acababan de finalizar sus estudios secundarios, con edades de en torno a 18-20 años, dedicados a tiempo completo a la actividad académica, y que dependían económicamente de sus familias. Actualmente, en la mayoría de los países, un alto porcentaje de quienes inician sus estudios universitarios no encajan en este perfil. La presencia de estudiantes no tradicionales en las instituciones universitarias se ha elevado en las últimas décadas, y se espera que continúe aumentando en los próximos años (Cruce y Williams, 2012; Klemencic y Fried, 2007). 
El concepto de estudiante no tradicional ha sido objeto de diferentes definiciones, basadas en la posesión de ciertas características que tradicionalmente no habían sido mayoritarias entre el alumnado universitario. Una primera forma común de caracterizarlos se basó en la edad con que se accede a la universidad, considerando como no tradicionales a quienes inician estudios con más de 23-25 años (Bean y Metzner, 1985). Este criterio, por sí solo, permite caracterizar como no tradicionales a un grupo heterogéneo de estudiantes. Se incluirían en él quienes han interrumpido sus estudios durante varios años, acceden a la universidad a través de vías alternativas a una titulación de nivel secundario, se encuentran ya desempeñando una actividad laboral o han constituido una familia. Horn (1996) establecía diferentes condiciones para caracterizar al estudiante no tradicional: inicia sus estudios universitarios años después de lo que hubiera podido hacerlo, se matricula de una parte de las materias del curso, trabaja a tiempo completo, es económicamente dependiente, tiene personas dependientes a su cargo, es padre o madre uniparental, o no posee un título de educación secundaria.

Más recientemente, la tipología de estudiantes no tradicionales se ha ampliado. Se incluye entre ellos a quienes se diferencian de la mayoría de los estudiantes por razones diversas: bajo nivel socioeconómico, formar parte de la primera generación que accede a la educación superior en sus familias, pertenecer a una minoría étnica o proceder de hogares donde la lengua materna difiere de la lengua utilizada en la universidad (Rendón, Jaromo y Nora, 2000). También se ha caracterizado como no tradicionales a los estudiantes que se desplazan a otros países para seguir estudios universitarios, o a los que proceden del medio rural y han de adaptarse a una cultura universitaria fundamentalmente urbana (Meuleman et al., 2014).

La investigación sobre el alumnado no tradicional se ha centrado frecuentemente en el análisis de su experiencia e integración en la vida universitaria, la compatibilización entre la condición de estudiantes y otros roles desempeñados al margen de la universidad, o las dificultades que encuentra para desenvolverse con éxito en los estudios, con especial atención a los problemas de bajo rendimiento y deserción (Bowl, 2001; Cantwell, Archer y Bourke, 2001; Munro, 2011; Schuetze y Slowey, 2002).

En el presente trabajo, consideraremos alumnado no tradicional al colectivo de estudiantes que compatibilizan estudios con otras responsabilidades laborales o familiares. Un rasgo característico del sistema universitario de Colombia, donde se contextualiza este traba- 
jo, es la alta presencia de alumnado joven que compatibiliza la realización de estudios con el desempeño de alguna actividad laboral. Pero a diferencia de lo que se observa en Europa o Estados Unidos, buena parte de este alumnado no ocupa puestos laborales de media jornada, sino de jornada completa, por lo que este rasgo es especialmente relevante para caracterizar al alumnado no tradicional en las universidades colombianas. Este tipo de alumnado colombiano no tradicional opta habitualmente por la jornada nocturna y encuentra importantes dificultades para finalizar una carrera universitaria (Buitrago, Fedossova y Britto, 2012).

\section{Objetivos e hipótesis}

Para Abbasi y Alghamdi (2015) la procrastinación constituye un complejo fenómeno psicológico que se encuentra entre los menos estudiados. En el mismo sentido, las revisiones de Steel (2007) y Rozental y Carlbring (2014) concluían que la procrastinación no ha sido aún suficientemente explicada. Este tipo de afirmaciones avalan la pertinencia de abordar estudios sobre el fenómeno de la procrastinación, que aporten nuevos resultados sobre las variables asociadas al mismo. A este hecho unimos la creciente presencia de alumnado no tradicional en la educación superior. La investigación al respecto ha mostrado que este tipo de estudiantes logra un menor rendimiento que el resto del alumnado (Cantwell, Archer y Bourke, 2001), y sus tasas de abandono tras el primer año son superiores a las que presentan los estudiantes tradicionales (Gilardi y Guglielmetti, 2011). De cara a la adopción de medidas para favorecer la integración en la universidad y el progreso académico, es interesante conocer si las conductas procrastinadoras están especialmente presentes en este colectivo estudiantil.

Como quedó reflejado en la introducción de este trabajo, la literatura ha prestado reiterada atención al papel de rasgos demográficos como el género, la edad, el estado civil o el nivel educativo en la explicación de las conductas procrastinadoras. Sin embargo, no hemos identificado hasta la fecha estudios que analicen una posible vinculación entre la procrastinación académica y la condición de estudiante no tradicional, caracterizando a éste en función de la combinación de sus estudios con otras ocupaciones. En este trabajo abordamos el análisis de las conductas de procrastinación académica, focalizando la atención en un colectivo de estudiantes colombianos no tradicionales. De acuerdo con ello, nos hemos planteado como objetivo analizar el nivel de procrastinación, el tipo de tareas que son procrastinadas y los motivos por los que procrastina el alumnado no tradicional, comparándolos con los correspondientes al alumnado tradicional. 
El alumnado no tradicional, caracterizado por simultanear los estudios universitarios con otras responsabilidades laborales o familiares, podría disponer de menos tiempo para el estudio. En consecuencia, nuestra hipótesis de trabajo es que este tipo de alumnado presenta mayores niveles de procrastinación que el alumnado tradicional.

\section{Método}

\section{Participantes}

Han participado en el estudio 479 estudiantes de las universidades colombianas El Bosque ( $n=393)$ y Uniempresarial ( $n=102)$, ambas de la ciudad de Bogotá, que cursaban programas de psicología, educación, ingenierías, y administración y negocios. Consideramos alumnado no tradicional a aquel que compatibiliza su actividad estudiantil con algún otro tipo de responsabilidades familiares o laborales que le impiden dedicarse al estudio a tiempo completo. A partir de la información aportada por los estudiantes se identificaron dos grupos de alumnado, caracterizados como tradicionales $(n=262)$ y no tradicionales $(n=217)$. En el grupo de estudiantes tradicionales, el 22,4\% son hombres y la media de edad se sitúa en 18,32 años $(\mathrm{DT}=2,75)$. Entre los no tradicionales, son hombres el $28,6 \%$ y la edad promedio alcanza los 20,52 años (DT=4,03).

\section{Instrumento}

Para valorar la procrastinación, recurrimos a la Procrastination Assessment Scale Students (PASS) elaborada por Solomon y Rothblum (1984), que se centra específicamente en la procrastinación académica en estudiantes universitarios. Hemos utilizado una versión española de este instrumento, adaptada para el contexto colombiano (Garzón y Gil, 2017). Un traductor profesional realizó una primera traducción de la PASS desde el inglés al español, que fue posteriormente revisada por tres psicólogos colombianos en busca de expresiones confusas o imprecisiones. Además, se aplicó la versión final a cinco estudiantes universitarios colombianos a quienes se les realizó una entrevista semiestructurada, al final de la aplicación de la prueba, en busca de términos confusos o ambiguos desde el punto de vista cultural. Al administrar la escala resultante, en el presente estudio se solicitó también a los participantes que completaran datos demográficos, incluyendo el género, la edad y la dedicación a los estudios (completa o compartida con otras responsabilidades laborales/familiares). 
La PASS consta de dos secciones, la primera de las cuales valora la prevalencia de procrastinación en seis tipos de tareas académicas: a) presentar un trabajo final, b) estudiar para un examen; c) llevar al día las lecturas asignadas semanalmente, d) realizar tareas administrativas (formalizar la matrícula, obtener el carnet universitario, etc.), e) asistir a reuniones (seminarios, tutorías, etc.) y f) desarrollar tareas académicas en general. En relación con cada una de ellas, los participantes debían indicar con qué frecuencia posponen la realización de dichas tareas hasta el último momento, de acuerdo con una escala Likert de cinco puntos: nunca (1), casi nunca (2), a veces (3), casi siempre (4) y siempre (5). Además, expresaron la frecuencia con que la demora de estas tareas representa un problema para ellos, usando la misma escala de respuesta. En la definición de procrastinación se incluye tanto el retraso en la realización de una tarea como la disconformidad con esa demora o la incomodidad que genera en el individuo. En consecuencia, la medida de procrastinación derivada de la PASS se obtiene sumando las dos puntuaciones obtenidas en frecuencia y carácter problemático de las demoras. De ese modo, resultan índices en una escala de valores comprendidos entre 2 y 10 .

La segunda sección de la PASS plantea al estudiante un supuesto de procrastinación (posponer la elaboración de un trabajo final), y le ofrece un abanico de 26 posibles razones que explicarían la procrastinación de esta tarea. Ansiedad, dificultades para tomar decisiones, baja asertividad, rebelión contra el control, miedo al éxito, aversión a la tarea o perfeccionismo, son algunos de los motivos sugeridos. Cada una de esas razones se formula a través de ítems o enunciados, para los cuales el estudiante debe indicar en qué grado reflejan la razón por la que procrastinó la última vez que demoró la redacción de un trabajo final. Se utiliza una escala Likert de cinco puntos, en la que el valor 1 corresponde a no refleja mis motivos en absoluto y 5 a los refleja perfectamente.

Las autoras de la PASS, Solomon y Rothblum (1984), no aportaron datos sobre su fiabilidad. No obstante, en un estudio posterior, Ferrari (1989) encontró una correlación testretest de $0,74(p<0,001)$. Las autoras sí informaron sobre la estructura factorial de los motivos para procrastinar, identificando dos factores principales. El primero agrupaba ansiedad, perfeccionismo y baja autoconfianza, explicando el 49,4\% de la varianza. El segundo incluía aversión a la tarea y pereza, y explicó el 18,0\% de la varianza. Otros factores aglutinaron el resto de las razones para procrastinar, pero fueron descartados por su escaso porcentaje de 
varianza explicada. En el presente estudio, los valores de alfa de Cronbach para la primera y segunda sección del instrumento se situaron en 0,86 y 0,91 respectivamente. Frente a los dos factores encontrados por Solomon y Rothblum (1984), el análisis factorial exploratorio de las razones para procrastinar detectó cinco factores que explican el 54\% de la varianza, con valores de alfa de Cronbach entre 0,71 y 0,82 en cada factor. Estos factores se identifican con búsqueda de excitación, falta de energía y autocontrol, perfeccionismo, ansiedad ante la evaluación, y falta de asertividad y confianza. En la Tabla 1 se muestran los ítems correspondientes a cada factor, sus pesos factoriales y los estadísticos descriptivos media y desviación típica. Se utilizó como método de extracción el análisis de componentes principales, seguido de una rotación mediante normalización varimax. En negrita se señala la asignación de cada ítem a uno de los cinco factores.

Tabla 1. Descriptivos y pesos factoriales de los ítems sobre motivos para procrastinar

\begin{tabular}{|c|c|c|c|c|c|c|c|}
\hline \multirow{2}{*}{ Factores / Ítems } & \multicolumn{5}{|c|}{ Componentes } & \multirow{2}{*}{ M } & \multirow{2}{*}{ (DT) } \\
\hline & 1 & 2 & 3 & 4 & 5 & & \\
\hline \multicolumn{8}{|l|}{ Búsqueda de excitación } \\
\hline - Te gustó el reto de esperar hasta la fecha de entrega & ,74 &, 04 &, 11 &, 01 &, 14 & 1,71 & $(1,06)$ \\
\hline $\begin{array}{l}\text { - Tenías ganas de sentir la emoción de hacer esta tarea en el } \\
\text { último momento }\end{array}$ & ,69 & 06 &,- 01 &, 10 &, 30 & 1,84 & $(1,12)$ \\
\hline $\begin{array}{l}\text { - Estabas preocupado de que si lo hacías bien, tus compañe- } \\
\text { ros te rechazaran }\end{array}$ & ,66 &, 01 &, 24 & ,04 &, 37 & 1,66 & $(1,00)$ \\
\hline - No te gustó que te pusieran plazos (fechas límites) & ,62 &, 32 &, 30 &, 15 &,- 06 & 1,89 & $(1,07)$ \\
\hline - No te gustó tener que hacer trabajos "mandados" por otros &, 59 &, 25 &, 16 & ,40 &,- 04 & 1,93 & $(1,16)$ \\
\hline \multicolumn{8}{|l|}{ Falta de energía y autocontrol } \\
\hline $\begin{array}{l}\text { - Te sentías demasiado perezoso para escribir un trabajo de } \\
\text { final de curso }\end{array}$ & ,09 & ,68 & ,30 &,- 11 &, 21 & 2,40 & $(1,22)$ \\
\hline - Tenías muchas otras cosas que hacer &,- 08 & ,65 &,- 25 & ,08 &, 05 & 2,81 & $(1,15)$ \\
\hline $\begin{array}{l}\text { - Sabías que tus compañeros tampoco habían empezado el } \\
\text { trabajo }\end{array}$ & ,44 &, 56 &, 12 & 09 &,- 11 & 2,17 & $(1,14)$ \\
\hline - No tenías bastante energía para empezar la tarea & 05 &, 55 & ,22 &,- 21 &, 38 & 2,46 & $(1,09)$ \\
\hline - No te gusta nada escribir trabajos extensos &, 17 &, 55 &, 15 &, 25 & ,28 & 2,32 & $(1,22)$ \\
\hline $\begin{array}{l}\text { - Esperaste hasta que un compañero hizo el suyo para que te } \\
\text { pudiera aconsejar }\end{array}$ & ,33 &, 52 &,- 03 & ,27 &, 25 & 2,05 & $(1,12)$ \\
\hline $\begin{array}{l}\text { - Pensabas que cuesta demasiado tiempo escribir un trabajo } \\
\text { de final de curso }\end{array}$ &, 16 & ,49 &, 39 &, 16 &, 15 & 2,36 & $(1,26)$ \\
\hline - Te sentías desbordado por la tarea &, 14 & ,48 &, 19 &, 31 & ,20 & 2,50 & $(1,15)$ \\
\hline - Tus amigos te presionaban para hacer otras cosas & ,38 & ,33 & ,32 &,- 06 &, 23 & 1,96 & $(1,12)$ \\
\hline $\begin{array}{l}\text { - Esperaste a ver si el profesor te ofrecía más información } \\
\text { sobre el trabajo }\end{array}$ &, 20 & ,31 & ,49 &, 24 & ,09 & 2,39 & $(1,15)$ \\
\hline \multicolumn{8}{|l|}{ Perfeccionismo } \\
\hline $\begin{array}{l}\text { - Te pusiste metas muy altas y te preocupaba no poder al- } \\
\text { canzarlas }\end{array}$ &, 12 &, 04 & ,75 & ,20 &, 16 & 2,69 & $(1,31)$ \\
\hline - Estabas preocupado de que si recibías una buena nota la & ,40 & 02 & ,64 & 09 &, 17 & 2,15 & $(1,27)$ \\
\hline
\end{tabular}


gente esperaría mucho de ti en el futuro

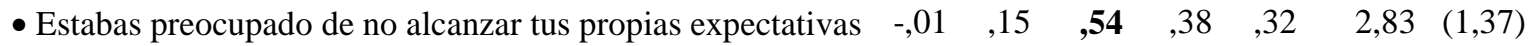

Ansiedad ante la evaluación

- Estabas preocupado de que al profesor no le gustara tu trabajo

23 - -,05 $\quad 15 \quad$,75 $\quad 18 \quad 2,57 \quad(1,23)$

- Estabas preocupado de recibir una mala calificación

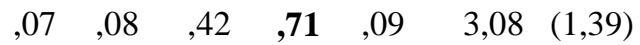

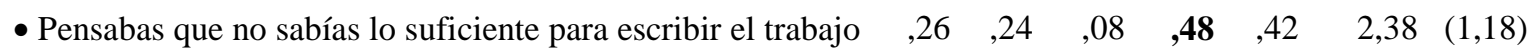
Falta de asertividad y confianza

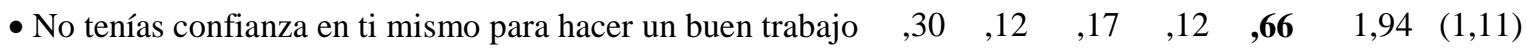

- Tenías problemas en pedir información a otros

29 $\quad$ 15 20 23 $\quad$,57 $2,12 \quad(1,09)$

- No podías elegir entre todos los posibles temas

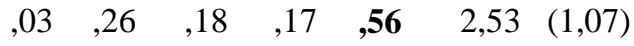

- Necesitabas pedir información al profesor pero no te sentías cómodo acercándote a el/ella

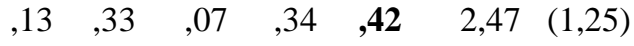

- Tenías dificultades en saber qué incluir y qué no incluir en tu trabajo

$\begin{array}{lllllll}-, 26 & , 34 & , 10 & , 47 & 32 & (1,03)\end{array}$

\section{Procedimiento}

La administración del PASS a los participantes en el estudio se realizó de manera colectiva, reuniéndolos en aulas de informática de sus respectivos centros. La prueba fue presentada y respondida a través de una encuesta informatizada, alojada en la página web de la Universidad El Bosque. Durante la aplicación, un investigador estuvo presente para aclarar posibles dudas de los participantes. Todos ellos fueron previamente informados sobre los objetivos del estudio y tomaron parte de manera voluntaria en la investigación. La confidencialidad de los datos recogidos les fue garantizada.

\section{Análisis de datos}

El análisis se ha iniciado con técnicas descriptivas, calculando los porcentajes de estudiantes tradicionales y no tradicionales que con alta frecuencia demoran la realización de las distintas tareas académicas y perciben que esa demora representa un problema. De manera separada en las dos submuestras de estudiantes, se han calculado medias y desviaciones típicas para los índices de procrastinación en las seis tareas que contempla la PASS y en un índice global definido como promedio de esos seis índices. Los mismos estadísticos han sido calculados para los motivos por los que el alumnado procrastina. En este caso, se han considerado los cinco factores identificados en la estructura factorial de la PASS, obteniendo la puntuación en cada uno de ellos como promedio de los ítems que subyacen a los mismos. La comparación de medias entre los dos grupos de alumnado se basa en la prueba t, calculando la d de Cohen para estimar el tamaño del efecto y valorar la magnitud de las diferencias observadas. 


\section{Resultados}

La tarea que con mayor frecuencia demora el alumnado no tradicional es la asistencia a reuniones, normalmente desarrolladas al margen del horario de clases (seminarios, tutorías), actividad que pospone siempre o casi siempre el $27,65 \%$ de estudiantes no tradicionales (Tabla 2). Destaca también la demora en llevar al día las lecturas asignadas semanalmente y realizar tareas administrativas, que afecta con frecuencia a más de la quinta parte de este colectivo. Comparativamente, el porcentaje de alumnado no tradicional que pospone tareas siempre o casi siempre supera al registrado para el alumnado tradicional en todas las tareas. En el caso de la presentación de un trabajo final, el porcentaje de estudiantes no tradicionales que se demoran frecuentemente llega a ser más del doble que el porcentaje registrado para el resto de estudiantes $(18,35 \%$ frente a 7,45\%). Ver Tabla 2.

Tabla 2. Porcentaje de alumnado que siempre o casi siempre pospone tareas y que percibe esta conducta como un problema

\begin{tabular}{lcccc}
\hline & \multicolumn{2}{c}{$\begin{array}{c}\text { Estudiantes } \\
\text { no tradicionales }\end{array}$} & $\begin{array}{c}\text { Estudiantes } \\
\text { Tareas }\end{array}$ & $\begin{array}{c}\text { Pospone } \\
\text { tareasionales }\end{array}$ \\
\cline { 2 - 5 } & $\begin{array}{c}\text { Posponer tareas } \\
\text { supone un } \\
\text { Problema }\end{array}$ & $\begin{array}{c}\text { Pospone } \\
\text { tareas }\end{array}$ & $\begin{array}{c}\text { Posponer tareas } \\
\text { supone un } \\
\text { problema }\end{array}$ \\
\hline Presentar un trabajo final & 19,35 & 43,26 & 7,45 & 38,43 \\
Estudiar para un examen & 19,82 & 41,40 & 11,76 & 32,55 \\
Llevar al día las lecturas asignadas & 23,04 & 41,47 & 14,90 & 33,33 \\
Realizar tareas administrativas & 22,12 & 32,26 & 13,73 & 20,78 \\
Asistir a reuniones (seminarios, tutorías,...) & 27,65 & 29,95 & 19,22 & 21,96 \\
Desarrollar tareas académicas en general & 14,75 & 24,88 & 9,80 & 19,61 \\
\hline
\end{tabular}

En lo que respecta a las tareas cuya demora representa siempre o casi siempre un problema para el alumnado no tradicional, destacan la presentación de un trabajo final $(43,26 \%)$, llevar al día las lecturas asignadas $(41,47)$ y estudiar para un examen $(41,40)$. Esas mismas tareas son también las que suponen mayor problema para el resto de los estudiantes. No obstante, es mayor el porcentaje de alumnado no tradicional que atribuye un carácter problemático a demorarse en su realización. La mayor diferencia se registra para la demora en realizar tareas administrativas. Esta situación es percibida siempre o casi siempre como un problema por el $32,26 \%$ de los estudiantes no tradicionales, cifra que se reduce al $20,78 \%$ en el otro 
grupo de estudiantes. Destacan también las diferencias obtenidas al valorar el carácter problemático atribuido a posponer tareas tales como estudiar para un examen, llevar al día las lecturas asignadas semanalmente o asistir a reuniones. En todos estos casos, las diferencias alcanzan o superan los ocho puntos porcentuales.

A diferencia de lo que ocurre en una demora activa o estratégica, las conductas procrastinadoras implican una demora que genera estrés para el individuo. Siguiendo las pautas proporcionadas por Solomon y Rothblum (1984), hemos sumado las puntuaciones obtenidas en la PASS para el grado de demora y el grado en que ésta representa un problema. Se generan así índices de procrastinación para cada tarea, comprendidos entre 2 y 10 . De acuerdo con este índice (Tabla 3), las conductas procrastinadoras afectan con mayor intensidad a estudiantes no tradicionales en la realización de un trabajo final (Media=6,13; D.T. $=1,55)$ y en llevar al día las lecturas asignadas semanalmente (Media=6,09; D.T.=1,72). En contraste con el resto de estudiantes, los no tradicionales muestran un índice global de procrastinación algo mayor, resultando significativas las diferencias $(t=4,412 ; \mathrm{p}<0,001 ; d=0,459)$. En lo que respecta a tareas concretas, los sobrepasan sobre todo al procrastinar tareas administrativas $(\mathrm{t}=4,048$; $\mathrm{p}<0,001 ; \mathrm{d}=0,374)$ o llevar al día las lecturas asignadas semanalmente $(\mathrm{t}=3,671 ; \mathrm{p}<0,001$; $\mathrm{d}=0,340$ ), aunque el tamaño de los efectos se mantiene en niveles discretos. Ver Tabla 3.

Tabla 3. Comparación de índices de procrastinación en tareas académicas entre estudiantes tradicionales y no tradicionales

\begin{tabular}{lccccc}
\hline \multirow{2}{*}{\multicolumn{1}{c}{ Índice de procrastinación }} & \multicolumn{3}{c}{ Media (Desv. Típica) } & & d de \\
\cline { 2 - 3 } & $\begin{array}{c}\text { Estudiantes no } \\
\text { tradicionales }\end{array}$ & $\begin{array}{c}\text { Estudiantes } \\
\text { tradicionales }\end{array}$ & & t & Cohen \\
\hline Presentar un trabajo final de curso & $6,13(1,55)$ & $5,66(1,48)$ & $3,337^{* * * *}$ & 0,310 \\
Estudiar para un examen & $5,85(1,85)$ & $5,41(1,68)$ & $2,666^{* * *}$ & 0,249 \\
Llevar al día las lecturas asignadas & $6,09(1,72)$ & $5,51(1,69)$ & $3,671^{* * * *}$ & 0,340 \\
Realizar tareas administrativas & $5,45(2,08)$ & $4,69(1,98)$ & $4,048^{* * *}$ & 0,374 \\
Asistir a reuniones (seminarios, tutorías,...) & $5,67(1,98)$ & $5,09(1,98)$ & $3,176^{* *}$ & 0,293 \\
Desarrollar tareas académicas en general & $5,35(1,78)$ & $5,04(1,56)$ & $1,998^{*}$ & 0,185 \\
\hline ÍNDICE GLOBAL (promedio en las 6 tareas) & $5,75(1,26)$ & $5,23(1,18)$ & $4,612^{* * *}$ & 0,459 \\
\hline${ }^{*} p<0,05 ;{ }^{* *} p<0,01 ;{ }^{* * *} p<0,001$ & & & & &
\end{tabular}

De acuerdo con los resultados mostrados en la Tabla 4, los motivos que en mayor medida justifican la procrastinación en el alumnado no tradicional son los que hacen referencia a la ansiedad ante la evaluación. En una escala de 5 puntos, el valor alcanzado por la media es Electronic Journal of Research in Educational Psychology, 15(3, 510-532. ISSN: 1696-2095. 2017. no. 43 
3,03. En cambio, la búsqueda de excitación es la causa menos relevante (media 1,83). Puntuaciones muy similares se observan para el alumnado tradicional, respecto al cual las diferencias en las puntuaciones medias no han resultado ser significativas.

Tabla 4. Motivos para procrastinar la realización de un trabajo final

\begin{tabular}{lcc}
\hline \multirow{1}{*}{ Motivos } & \multicolumn{2}{c}{ Media (Desv, típica) } \\
\cline { 2 - 3 } & $\begin{array}{c}\text { Estudiantes } \\
\text { no tradicionales }\end{array}$ & $\begin{array}{c}\text { Estudiantes } \\
\text { tradicionales }\end{array}$ \\
\hline Búsqueda de excitación & $1,83(0,82)$ & $1,79(0,79)$ \\
Falta de energía y autocontrol & $2,40(0,75)$ & $2,28(0,68)$ \\
Perfeccionismo & $2,54(1,04)$ & $2,57(1,05)$ \\
Ansiedad ante la evaluación & $3,03(0,91)$ & $2,91(0,85)$ \\
Poca asertividad y confianza & $2,46(0,82)$ & $2,42(0,70)$ \\
\hline
\end{tabular}

\section{Discusión y conclusiones}

Los resultados del presente estudio han mostrado que el alumnado no tradicional supera en niveles de procrastinación académica a sus compañeros o compañeras que encajan en un perfil tradicional de estudiante. Se confirma por tanto nuestra hipótesis de partida, si bien el tamaño del efecto para la diferencia de medias observada entre ambos colectivos es mediobajo. En cuanto al tipo de tareas que son procrastinadas, se encuentra un paralelismo entre los dos tipos de estudiantes, ya que las tareas más demoradas en ambos grupos son coincidentes. Las tareas más procrastinadas son la entrega de trabajos finales y las lecturas asignadas semanalmente. Es en estas últimas, y sobre todo en las tareas administrativas, donde los estudiantes no tradicionales superan con mayor claridad a los tradicionales en niveles de procrastinación. De acuerdo con ello, encontrar el tiempo necesario para cubrir trámites ante servicios administrativos, a menudo con rígidos horarios de atención al alumnado, representa un problema especialmente para quienes han de atender a responsabilidades familiares o laborales. Pero los problemas de procrastinación también diferencian a ambos tipos de estudiantes en lo que respecta a tareas académicas propiamente dichas. Para su desarrollo, la menor disponibilidad de tiempo supone un claro obstáculo que deben salvar quienes cuentan con otras ocupaciones. Manthei y Gilmore (2005) encontraron que precisamente la falta de tiempo es percibida por los estudiantes como un inconveniente derivado de trabajar a tiempo parcial, y el problema se agrava cuando se trabaja en jornadas completas (Choy, 2002). 
Estudios anteriores han puesto de relieve que la ocupación del alumnado en responsabilidades ajenas a la universidad conlleva algunas ventajas: incremento de las habilidades de comunicación, relación con otras personas, autoconfianza, y también una menor tendencia que en otros estudiantes a perder el tiempo, junto con un mayor desarrollo de las habilidades para gestionarlo (Robotham, 2012). En esta línea cabría esperar que el alumnado no tradicional, identificado fundamentalmente por sus responsabilidades extraacadémicas, se situara en una posición favorable para evitar conductas procrastinadoras. Sin embargo, los resultados que hemos obtenido no encajan plenamente en este escenario. Aunque la ocupación del alumnado en otras actividades acarrea efectos positivos, podríamos hipotetizar que una dedicación elevada de tiempo a otras tareas reduce severamente la disponibilidad de horas para el estudio, poniendo en riesgo los beneficios esperados de la experiencia laboral. Téngase en cuenta que, en la muestra utilizada para este estudio, el alumnado no tradicional que trabaja lo hace en jornadas con una duración elevada, mientras que la investigación previa ha venido ocupándose de alumnado que compatibiliza la universidad con un empleo a tiempo parcial.

La existencia de una relación negativa entre procrastinación y rendimiento académico ha sido suficientemente ilustrada en el reciente meta-análisis de Kim y Seo (2015). Con posterioridad, Grunschel, Schwinger, Steinmayr y Fries (2016) han corroborado que un aumento en el uso de estrategias de autorregulación, decrementa la procrastinación y aumenta el rendimiento. De esta relación deriva la necesidad de una intervención preventiva sobre el alumnado procrastinador, que contribuya a mejorar sus expectativas de éxito en la universidad. Teniendo en cuenta que los modelos teóricos actuales sobre la autorregulación ayudan a entender la procrastinación como un tipo de comportamiento desregulatorio (de la Fuente, 2017), una dirección en la que intervenir apunta a incrementar el uso de estrategias de autorregulación.

En nuestro estudio hemos constatado una tendencia a mayor procrastinación en el alumnado no tradicional. Ello subraya su condición de población de riesgo, susceptible de verse afectada por problemas de fracaso o abandono. Aunque las distancias respecto al resto de estudiantes no son grandes, parece aconsejable que las actuaciones dirigidas al alumnado procrastinador presten especial atención a los estudiantes no tradicionales. Y más aún ante la previsión de un incremento de este alumnado en los próximos años a nivel internacional (Klemencic y Fried, 2007). 
Es cierto que las políticas educativas han venido favoreciendo la incorporación a la universidad de sectores amplios de la población, incrementando la presencia de alumnado que se aleja del perfil tradicional. En el caso de América Latina y el Caribe, entre 2000 y 2010 la cobertura universitaria aumentó en un 40\%, lo que indica que cada vez más se están incorporando estudiantes con perfiles diversos. Paradójicamente, el sistema universitario no se ha adaptado lo suficiente a las características y necesidades de este tipo de estudiantes (Gilardi; Guglielmetti, 2011). El necesario apoyo al alumnado no tradicional habría de incluir un tratamiento adecuado de las conductas procrastinadoras, que con mayor intensidad afectan a este colectivo que al resto de estudiantes.

Más allá de los libros de autoayuda que han venido apareciendo en el ámbito anglosajón (Burka y Yuen, 2008; Ferrari, 2010; Steel, 2012), la procrastinación habría de ser objeto de las actuaciones desarrolladas en el ámbito de la orientación académica por parte de los servicios universitarios de orientación. Se han propuesto diferentes estrategias para el tratamiento de la procrastinación, haciendo hincapié en la identificación de las causas, el análisis y control de los elementos distractores que compiten con las tareas académicas, la modificación de concepciones distorsionadas que las alimentan (por ejemplo, ansiedad ante la evaluación, miedo al fracaso, perfeccionismo, excesivo optimismo, baja autoeficacia, baja autoestima), o la reducción de los sentimientos negativos que genera (Abbasi y Alghamdi, 2015; Balkis y Duru, 2007, 2017; Schouwenburg et al., 2004). Son prometedoras las intervenciones flexibles para el tratamiento de la procrastinación, usando entrenamiento no presencial (Glick y Orsillo 2015; Rozental et al., 2014), así como la incorporación de tutorías virtuales por parte de los docentes o la atención administrativa mediante chat o vías no convencionales.

De acuerdo con Klingsieck (2013), la intervención orientadora sobre las conductas de procrastinación debería asumir un enfoque personalizado, adaptado al perfil de cada estudiante y basado en los motivos por los que procrastina. Para el tipo de alumnado no tradicional al que corresponden los participantes en este trabajo, la disponibilidad de tiempo para el estudio es menor. Por ello, la intervención orientadora habría de proporcionar también ayuda para optimizar la gestión del tiempo a través de pautas tales como fijar metas y objetivos realistas, establecer prioridades, y utilizar herramientas para la planificación temporal de tareas y para el seguimiento de su ejecución. 
Como en otros trabajos empíricos, los resultados obtenidos habrían de tomarse con cautela, debido a las limitaciones inherentes a la investigación. En primer lugar, dado que la definición de estudiante no tradicional no es única y depende del contexto (Kim, 2002), los resultados obtenidos no son directamente generalizables a cualquier tipo de alumnado no tradicional. Además el estudio adolece de una debilidad propia de los procedimientos basados en el autoinforme, que conlleva el riesgo de sesgo en la información aportada por los participantes. No obstante, esta limitación podría tener menos peso teniendo en cuenta los resultados del estudio de Krause y Freund (2014), quienes han demostrado que las medidas basadas en el autoinforme de los estudiantes reflejan más adecuadamente el constructo procrastinación académica que su medición a partir de la observación directa de las conductas dilatorias en el trabajo académico.

En respuesta a la primera de las limitaciones que afectan al presente estudio, una línea para futuras investigaciones iría en la dirección de abordar el problema de la procrastinación en una mayor variedad de instituciones y valorando diferentes perfiles de alumnado no tradicional. Otro ámbito interesante de estudio se sitúa en la implementación y evaluación de intervenciones orientadoras para el tratamiento de la procrastinación académica, especialmente las dirigidas al alumnado no tradicional de nuevo ingreso.

\section{Referencias}

Abbasi, I.S. and Alghamdi, N.G. (2015). The prevalence, predictors, causes, treatments, and implications of procrastination behaviors in general, academic, and work setting. International Journal of Psychological Studies, 7(1), 59-66. doi:10.5539/ijps.v7n1p59

Balkis, M., and Duru, E. (2007). The evaluation of the major characteristics and aspects of the procrastination in the framework of psychological counseling and guidance. Educational Sciences: Theory \& Practice, 7(1), 376-385.

Balkis, M., and Duru, E. (2017). Gender differences in the relationship between academic procrastination, satisfaction with academic life and academic performance. Electronic Journal of Research in Educational Psychology 15(1), 105-125. doi: 10.14204/ejrep.41.16042

Bean, J.P. and Metzner, B.S. (1985). A conceptual model of nontraditional undergraduate student attrition. Review of Educational Research, 55(4), 485-540. doi:10.3102/00346543055004485 
Bowl, M. (2001). Experiencing the barriers: non-traditional students entering Higher Education. Research Papers in Education, 16(2), 141-160. doi:10.1080/02671520110037410

Buitrago, O., Fedossova, A. y Britto, R. (2012). Inferencias para la reflexión sobre la calidad de la educación superior nocturna en ingeniería en Colombia. Educación y Educadores, 15(3), 431-443. doi:10.5294/edu.2012.15.3.5

Burka, J.B. and Yuen, L.M. (2008). Procrastination: why you do it, what to do about now? New York, NY: Da Capo Lifelong Books.

Cantwell, R., Archer, J. and Bourke, S. (2001). A comparison of the academic experiences and achievement of university student entering by traditional and nontraditional means. Assessment \& Evaluation in Higher Education, 26(3), 221-234. doi:10.1080/02602930120052387

Choy, S. (2002). Nontraditional undergraduates. Washington, DC: National Center for Education Statistics.

Corkin, D.M., Yu, S.L. and Lindt, S.F. (2011). Comparing active delay and procrastination from a self-regulated learning perspective. Learning and Individual Differences, 21, 602-606. doi:10.1016/j.lindif.2011.07.005.

Cruce, T. and Williams, N. (2012). Preparing for the silver tsunami: the demand for Higher Education among older adults. Research in Higher Education, 53(6), 593-613. doi:10.1007/s11162-011-9249-9

Day, V., Mensink, D. and O'Sullivan, M. (2000). Patterns of academic procrastination. Journal of College Reading and Learning, 30, 120-134. doi:10.1080/10790195.2000.10850090

De la Fuente-Arias, J. (2017). Theory of Self- vs. Externally-Regulated Learning ${ }^{\mathrm{TM}}$ : fundamentals, evidence, and applicability. Frontiers in Psychology, 8, 1675. doi:10.3389/fpsyg.2017.01675

Ferrari, J. R. (1989). Reliability of academic and dispositional measures of procrastination. Psychological Reports, 64(3c), 1057-1058. doi:10.2466/pr0.1989.64.3c.1057

Ferrari, J.R. (2010). Still procrastinating? The no-regrets guide to getting it done. Hoboken, New Jersey: Wiley.

Ferrari, J. R. and Díaz-Morales, J.F. (2014). Procrastination and mental health coping: a brief report related to students. Individual Differences Research, 12(1), 8-11.

Ferrari, J. R. and Scher, S.J. (2000). Toward an Understanding of Academic and Nonacademic Tasks Procrastinated by Students: The Use of Daily Logs. Psychology in the 
Schools, $\quad 37, \quad 359-366 . \quad$ doi:10.1002/1520-6807(200007)37:4<367::AIDPITS7>3.0.CO;2-Y

Garzón, A. y Gil, J. (2017). Propiedades psicométricas de la versión en español de la prueba PASS. Revista Iberoamericana de Diagnóstico y Evaluación Psicológica, 43(1), 149163. doi:10.21865/RIDEP43_149

Gilardi, S. and Guglielmetti, C. (2011). University life of non-traditional students: engagement styles and impact on attrition. The Journal of Higher Education, 82(1), 33-53. doi:10.1353/jhe.2011.0005

Glick, D.M., and Orsillo, S.M. (2015). An investigation of the efficacy of acceptance-based behavioral therapy for academic procrastination. Journal of Experimental Psychology: General, 144(2), 400. doi:10.1037/xge0000050

Grunschel, C., Schwinger, M., Steinmayr, R., and Fries, S. (2016). Effects of using motivational regulation strategies on students' academic procrastination, academic performance, and well-being. Learning and Individual Differences, 49, 162-170. doi: 10.1016/j.lindif.2016.06.008

Gupta, R., Hershey, D. and Gaur, J. (2012). Time perspective and procrastination in the workplace: an empirical investigation. Current Psychology, 31(2), 195-211. doi:10.1007/s12144-012-9136-3

Higgins, G.E. and Tewksbury, R. (2006). Sex and self-control theory: the measures and causal model may be different. Youth Society, 37(4), 479-503. doi:10.1177/0044118x05283423

Horn, L. (1996). Nontraditional undergraduates, trends in enrollment from 1986 to 1992 and persistence and attainment among 1989-90 beginning postsecondary students. Washington, DC: National Center for Education Statistics.

Kim, K.R. and Seo, E.H. (2015). The relationship between procrastination and academic performance: a meta-analysis. Personality and Individual Differences, 82, 26-33. doi:10.1016/j.paid.2015.02.038

Kim, K.R. (2007). ERIC Review: Exploring the meaning of "nontraditional" at the community college. Community College Review, 30(1), 74-88. doi:10.1177/009155210203000104

Klemencic, M. and Fried, J. (2015). Demographic challenges and future of the Higher Education. International Higher Education, 47, 12-14. 
Klingsieck, K.B. (2013). Procrastination. European Psychologist, 18(1), 24-34. doi:10.1027/1016-9040/a000138

Krause, K. and Freund, A.M. (2014). Delay or procrastination - A comparison of self-report and behavioral measures of procrastination and their impact on affective well-being. Personality and Individual Differences, 63, 75-80. doi:10.1016/j.paid.2014.01.050

Manthei, R.J. and Gilmore, A. (2005). The effect of paid employment on university students' lives. Education+ Training, 47(3), 202-215. doi:10.1108/00400910510592248

Meuleman, A.M., Garrett, R., Wrench, A. and King, S. (2014). 'Some people might say I'm thriving but...': non-traditional students' experiences of university. International Journal of Inclusive Education,19(5), 503-517. doi:10.1080/13603116.2014.945973

Munro, L. (2011). 'Go boldly, dream large!': The challenges confronting non-traditional students at university. Australian Journal of Education, 55(2), 115-131. doi:10.1177/000494411105500203

Onwuegbuzie, A. (2004). Academic procrastination and statistics anxiety. Assessment y Evaluation in Higher Education, 29(1), 3-19. doi:10.1080/0260293042000160384

Pychyl, T.A. and Flett, G.L. (2012). Procrastination and self-regulatory failure: an introduction to the special issue. Journal of Rational-Emotive \& Cognitive-Behavior Therapy, 30, 203-212. doi:10.1007/s10942-012-0149-5

Rendón, L.I., Jalomo, R.E. and Nora, A. (2000). Theoretical considerations in the study of minority student retention in higher education. En J.M. Braxton (Ed.), Reworking the student departure puzzle (pp. 127-156). Nashville, TN: Vanderbilt University Press.

Robotham, D. (2012). Student part-time employment: characteristics and consequences, $E d u$ cation + Training, 54(1), 65 - 75. doi:10.1108/00400911211198904

Rozental, A. and Carlbring, P. (2013). Internet-based cognitive behavior therapy for procrastination: Study protocol for a randomized controlled trial. Journal of Medical Internet Research, 15(11), 27. doi:10.2196/resprot.2801

Rozental, A. and Carlbring, P. (2014). Understanding and treating procrastination: a review of a common self-regulatory failure. Psychology, 5(13), 1488-1502. doi:10.4236/psych.2014.513160

Rozental, A., Forsström, D., Nilsson, S., Rizzo, A. and Carlbring, P. (2014). Group versus Internet-based cognitive-behavioral therapy for procrastination: study protocol for a randomized controlled trial. Internet Interventions, 1(2), 84-89. doi:10.1016/j.invent.2014.05.005 
Schouwenburg, H.C., Lay, C. H., Pychyl, T.A. and Ferrari, J.R. (Eds.). (2004). Counseling the procrastinator in academic settings. Washington, DC: American Psychological Association.

Schraw, G., Wadkins, T. and Olafson, L. (2007). Doing the things we do: A grounded theory of academic procrastination. Journal of Educational Psychology, vol.99 (1), 12-25, doi:10.1037/0022-0663.99.1.12

Schuetze, H.G. and Slowey, M. (2002). Participation and exclusion: a comparative analysis of non-traditional students and lifelong learners in higher education. Higher Education, 44(3-4), 309-327.

Sirois, F.M. (2004). Procrastination and intentions to perform health behaviors: The role of self-efficacy and the consideration of future consequences. Personality and Individual Differences, 37 (1), 115-128. http://dx.doi.org/10.1016/j.paid.2003.08.005

Solomon, L. and Rothblum, E. (1984). Academic Procrastination: Frequency and CognitiveBehavioral Correlates. Journal of Couseling Psychology, 31(4), 503-509. doi:10.1037/0022-0167.31.4.503

Steel, P. (2007). The nature of procrastination: a meta-analytic and theoretical review of quintessential self regulatory failure. Psychological Bulletin 133, 65-94. doi:10.1037/00332909.133.1.65

Steel, P. (2012). The procrastination equation. how to stop putting things off and start getting things done. Edinburgh: Pearson Education Ltd.

Steel, P. and Ferrari, J. (2013). Sex, education and procrastination: an epidemiological study of procrastinators' characteristics from a global sample. European Journal of Personality, 27(1), 51-58. doi:10.1002/per.1851

Strüber, D., Lück, M. and Roth, G. (2008). Sex, aggression and impulse control: an integrative account. Neurocase: The Neural Basis of Cognition, 14(1), 93-121. doi:10.1080/13554790801992743

Strunk, K., Cho, Y., Steele, M. and Bridges, S. (2013) Development and validation of a $2 \times 2$ model of time-related academic behavior: procrastination and timely engagement. Learning and individual differences, 25, 35-44. doi:10.1016/j.lindif.2013.02.007 